\title{
DRINFELD-SOKOLOV REDUCTION FOR DIFFERENCE OPERATORS AND DEFORMATIONS OF $W$-ALGEBRAS. II. GENERAL SEMISIMPLE CASE
}

\author{
M. A. SEMENOV-TIAN-SHANSKY AND A. V. SEVOSTYANOV
}

April 10, 1994

\begin{abstract}
The paper is the sequel to [9]. We extend the Drinfeld-Sokolov reduction procedure to q-difference operators associated with arbitrary semisimple Lie algebras. This leads to a new elliptic deformation of the Lie bialgebra structure on the associated loop algebra. The related classical r-matrix is explicitly described in terms of the Coxeter transformation. We also present a cross-section theorem for q-gauge transformations which generalizes a theorem due to R.Steinberg.
\end{abstract}

\section{INTRODUCTION}

The present paper is the sequel to [9]; we refer the reader to this paper for a general introduction. Our goal is to extend the results of [9] to arbitrary semisimple Lie algebras. As an intermediate step we develop a group-theoretic framework for abstract difference equations associated with arbitrary semisimple Lie groups. A similar problem for differential equations which was solved by Drinfeld and Sokolov is linear, since it involves only the structure of the corresponding semisimple Lie algebra. Difference equations lead to the study of specific submanifolds in Lie groups which are closely related to some of its Bruhat cells. Our main technical result is a cross-section theorem for the q-gauge transformations which generalizes a theorem due to R.Steinberg. The reduction scheme outlined in [9] extends to the abstract case as well and the general conclusion remains the same: the consistency condition for the reduction imposes very rigid conditions on the underlying classical r-matrix which fix it completely. The resulting classical r-matrix is new; it yields an elliptic deformation of the Lie bialgebra structure on the loop algebras associated with the so called Drinfeld's new realizations of quantized affine algebras [3], [10]. The explicit characterization of the set of abstract q-difference operators leads to a very simple formula for this r-matrix in terms of the Coxeter element of the corresponding Weyl group. (The role of Coxeter transformations in the theory of q-difference operators should be compared with the role of the dual Coxeter transformations in the representation theory of affine Lie algebras at the critical level which is implicit in [7], [8]). The Coxeter element also plays a key role in the definition and the study of the generalized Miura transformation. 
The structure of the paper is as follows. Section 1 gives a description of the set of abstract q-difference operators associated to an arbitrary complex semisimple Lie group $G$. This description is based on the cross-section theorem referred to above; its proof is postponed until section 3. In section 2 we define a class of Poisson covariant Poisson structures on the set of q-difference $G$-valued connections; this definition is preceded by the description of a class of Lie bialgebra structures on loop algebras. As compared to [9], we need more details on the Poisson theory of q-gauge transformations, including the theory of the double and the twisted factorization. We then formulate our main theorem which gives an explicit description of the (unique) classical r-matrix which is compatible with the Drinfeld-Sokolov reduction for qdifference operators associated with $G$. At the end of section 2 we also briefly discuss the reduction for finite difference operators on the lattice which yields a definition of the classical lattice $W(\mathfrak{g})$-algebra extending the definition of the lattice Virasoro algebra discussed in [9]. Section 4 contains a description of the generalized Miura transformations. Finally, in section 5 we compare our formulae with the formulae of Frenkel and Reshetikhin [8] for the $\operatorname{sl}(n)$ case.

Acknowledgements. The present paper is part of a joint research project which was started by E.Frenkel and N.Reshetikhin together with the first author. We are indebted to B.Kostant who has pointed us the paper [16]; one of the authors (M.S.T.S) would like to thank G.Arutyunov for useful discussion.

\section{AbStract Q-DifFEREnCE OPERATORS}

The following notation will be used throughout the paper. Let $G$ be a connected simply connected finite-dimensional complex semisimple Lie group, $\mathfrak{g}$ its Lie algebra. Fix a Cartan subalgebra $\mathfrak{h} \subset \mathfrak{g}$ and let $\Delta$ be the set of roots of $(\mathfrak{g}, \mathfrak{h})$. Choose an ordering in the root system; let $\Delta_{+}$be the system of positive roots and $\left\{\alpha_{1}, \ldots, \alpha_{l}\right\}$, $l=\operatorname{rank} \mathfrak{g}$, the corresponding set of simple roots. For $\alpha \in \Delta, \alpha=\sum_{i=1}^{l} n_{i} \alpha_{i}$ we define its height by

$$
\text { height } \alpha=\sum_{i=1}^{l} n_{i} \text {. }
$$

Let $e_{\alpha} \in \mathfrak{g}$ be a root vector which corresponds to $\alpha \in \Delta$. Let

$$
\mathfrak{b}=\mathfrak{h}+\sum_{\alpha \in \Delta_{+}} \mathbb{C} e_{\alpha}
$$

be the corresponding Borel subalgebra and

$$
\overline{\mathfrak{b}}=\mathfrak{h}+\sum_{\alpha \in \Delta_{+}} \mathbb{C} e_{-\alpha}
$$

the opposite Borel subalgebra; let $\mathfrak{n}=[\mathfrak{b}, \mathfrak{b}]$ and $\overline{\mathfrak{n}}=[\overline{\mathfrak{b}}, \overline{\mathfrak{b}}]$ be their respective nilradicals. We shall fix a nondegenerate invariant bilinear form $($,$) on \mathfrak{g}$. Let $H=$ $\exp \mathfrak{h}, N=\exp \mathfrak{n}, \bar{N}=\exp \overline{\mathfrak{n}}, B=H N, \bar{B}=H \bar{N}$ be the Cartan subgroup, the 
maximal unipotent subgroups and the Borel subgroups of $G$ which correspond to the Lie subalgebras $\mathfrak{h}, \mathfrak{n}, \overline{\mathfrak{n}}, \mathfrak{b}$ and $\overline{\mathfrak{b}}$, respectively.

Let $W$ be the Weyl group of $(\mathfrak{g}, \mathfrak{h})$; we shall denote a representative of $w \in W$ in $G$ by the same letter. Let $s_{1}, \ldots, s_{l}$ be the reflections which correspond to simple roots; let $s=s_{1} s_{2} \cdots s_{l}$ be a Coxeter element. Put $N^{\prime}=\left\{v \in N ; s v s^{-1} \in \bar{N}\right\}$; it is easy to see that $N^{\prime} \subset N$ is an abelian subgroup, $\operatorname{dim} N^{s}=l$. Put $M^{s}=N s^{-1} N$; it is clear that $N s^{-1} N=N^{\prime} s^{-1} N$ and that $N^{\prime} \times N \rightarrow M^{s}:\left(n^{\prime}, n\right) \longmapsto n^{\prime} s^{-1} n$ is a diffeomorphism.

Let $\mathcal{G}=L G$ be the loop group of $G$; the group $G$ will be identified with the subgroup of constant loops in $\mathcal{G}$. Fix $q \in \mathbb{C},|q|<1$, and let $\tau$ be the automorphism of $\mathcal{G}$ defined by $g^{\tau}(z)=g(q z)$. We shall denote the corresponding automorphism of the loop algebra $L \mathfrak{g}$ by the same letter. Let $\mathcal{C}$ be another copy of $\mathcal{G}$ equipped with the q-gauge action of $\mathcal{G}$,

$$
\mathcal{G} \times \mathcal{C} \rightarrow \mathcal{C} ;(v, L) \longmapsto v^{\tau} L v^{-1}
$$

The space $\mathcal{C}$ will be sometimes referred to as the space of q-difference connections (with values in $G$ ). Let $\mathcal{M}^{s}$ be the cell in $\mathcal{C}$ consisting of loops with values in $M^{s}$, and $\mathcal{S}=\mathcal{N}^{\prime} s^{-1}$

Theorem 1.1. The restriction of the gauge action (1.1) to $\mathcal{N} \subset \mathcal{G}$ leaves the cell $\mathcal{M}^{s} \subset \mathcal{C}$ invariant. The action of $\mathcal{N}$ on $\mathcal{M}^{s}$ is free and $\mathcal{S}$ is a cross-section of this action.

The proof will be given in section 3. (Its special case which corresponds to $G=$ $S L(n)$ is presented in [9].)

Remark. A closely related theorem is due to Steinberg [16] who discussed the action of an algebraic semisimple Lie group on itself by conjugations (In other words, the situation considered in [16] corresponds to the trivial automorphism $\tau=i d$.) Steinberg's theorem asserts that if $G$ is defined over an algebraically closed field, $N^{\prime} s^{-1} \subset G$ is a cross-section of the set of regular conjugacy classes in $G$. In theorem 1.1 we replace the action of the entire group on itself by the action of its unipotent subgroup on its affine subvariety $\mathcal{M}^{s}$. In the context of Lie algebras a similar problem was studied by B.Kostant [11], again in the case of trivial $\tau$. $\square$

To motivate the above definitions let us discuss the case $G=S L(n)$. Let us choose an order in the root system of $\mathfrak{s l}(n)$ in such a way that positive root vectors correspond to lower triangular matrices. We may order the simple roots and choose the matrices $s_{k}, k=1, \ldots, n-1$, representing the corresponding reflections in such a way that the 
Coxeter element $s=s_{1} s_{2} \ldots s_{n-1}$ is represented by the matrix

$$
s=\left(\begin{array}{ccccc}
0 & 0 & \cdots & 0 & 1 \\
-1 & 0 & \cdots & 0 & 0 \\
0 & -1 & \cdots & 0 & 0 \\
\cdots & \cdots & \cdots & \cdots & \cdots \\
0 & 0 & \cdots & 0 & 0 \\
0 & 0 & \cdots & -1 & 0
\end{array}\right)
$$

Then the group $N^{\prime}$ consists of lower triangular unipotent matrices of the form

$$
u=\left(\begin{array}{ccccc}
1 & 0 & \ldots & 0 & 0 \\
0 & 1 & \ldots & 0 & 0 \\
\ldots & \ldots & \ldots & \ldots & \ldots \\
0 & 0 & \ldots & 1 & 0 \\
* & * & \cdots & * & 1
\end{array}\right)
$$

the set $M^{s}$ consists of all unimodular matrices of the form

$$
A=\left(\begin{array}{ccccc}
* & -1 & 0 & \ldots & 0 \\
* & * & -1 & \ldots & 0 \\
\ldots & \ldots & \ldots & \ldots & \ldots \\
* & * & * & \ldots & -1 \\
* & * & * & \ldots & *
\end{array}\right)
$$

and the set $N^{\prime} s^{-1}$ is the set of all companion matrices of the form

$$
L=\left(\begin{array}{cccccc}
0 & -1 & & \cdots & 0 & 0 \\
0 & 0 & -1 & \cdots & 0 & 0 \\
0 & 0 & 0 & \cdots & 0 & 0 \\
\cdots & \cdots & \cdots & \cdots & \cdots & \cdots \\
0 & 0 & 0 & \cdots & 0 & -1 \\
1 & u_{1} & u_{2} & \cdots & u_{n-2} & u_{n-1}
\end{array}\right) .
$$

Let us associate to $L$ a first order difference equation

$$
\tau \cdot \psi+L \psi=0
$$

where $\psi=\left(\psi_{1}, \psi_{2}, \ldots, \psi_{n}\right)^{t}$ is a column vector, $\psi_{k} \in \mathbb{C}((z))$. It is easy to see that its first component $\phi:=\psi_{1}$ satisfies an $n$-th order difference equation,

$$
\tau^{n} \phi+u_{n-1} \tau^{n-1} \cdot \phi+u_{n-2} \tau^{n-2} \cdot \phi+\ldots+u_{1} \tau \cdot \phi+\phi=0
$$

and, moreover, $\psi_{k}=\tau^{k-1} \phi, k=1,2, \ldots, n$. Hence the set $\mathcal{N}^{\prime} s^{-1}$ may be identified with the set $\mathcal{M}_{n, q}$ of all $\mathrm{n}$-th order q-difference operators. ${ }^{1}$ In the general case we set, by

\footnotetext{
${ }^{1}$ Note that in this paper we use slightly different conventions, as compared to [9]; in particular, we consider the q-gauge action by lower triangular matrices as opposed to upper triangular matrices
} 
definition, $\mathcal{M}_{q}(G)=\mathcal{M}^{s} / L N$; as we shall see, the manifold $\mathcal{M}_{q}(G)$ carries a natural Poisson structure and may be regarded as the spectrum of a classical q-W-algebra.

Remark. In [4] Drinfeld and Sokolov use a similar approach to define the set of abstract higher order differential operators associated to a given semisimple Lie algebra; the key observation that motivates their definition is that for $\mathfrak{g}=\mathfrak{s l}(n)$ the matrix

$$
f=\left(\begin{array}{cccccc}
0 & -1 & 0 & \cdots & 0 & 0 \\
0 & 0 & -1 & \cdots & 0 & 0 \\
\cdots & \cdots & \cdots & \cdots & \cdots & \cdots \\
0 & 0 & 0 & \cdots & 0 & -1 \\
0 & 0 & 0 & \cdots & 0 & 0
\end{array}\right) \in \mathfrak{g}
$$

is a principal nilpotent element. Accordingly, in the context of Lie algebras the set $\mathcal{M}^{s} \subset \mathcal{G}$ is replaced by the affine manifold $\mathcal{M}^{f}=f+L \mathfrak{b} \subset L \mathfrak{g}$; the main cross-section theorem then asserts that the gauge action of the subgroup $L N \subset L G$ leaves $\mathcal{M}^{f}$ invariant; its restriction to $\mathcal{M}^{f}$ is free and admits a cross-section which is an affine submanifold in $L \mathfrak{g}$. This cross-section provides a model for the space of higher order differential operators. One may notice that the 'near coincidence' of the matrices $s^{-1}$ and $f$ is peculiar to the $S L(n)$ case.

\section{Poisson Structures for Q-Difference EQuations}

In this section we shall construct a class of Poisson structures on $\mathcal{G}=L G$ and on the space $\mathcal{C}$ of q-difference connections. Our main theorem asserts that there is a unique Poisson structure in this class which is compatible with the Poisson reduction over $L N$. We start with the description of a family of Lie bialgebra structures on loop algebras.

2.1. Factorizable Lie bialgebras associated with $L \mathfrak{g}$. Let $g=L \mathfrak{g}$ be the loop algebra; we equip it with the standard invariant bilinear form,

$$
\langle X, Y\rangle=\operatorname{Res}_{z=0}(X(z), Y(z)) d z / z .
$$

Notice that the automorphism $\tau$ satisfies $\langle\tau X, \tau Y\rangle=\langle X, Y\rangle$. Let $d=g \oplus g$ be the direct sum of two copies of $g$ with the bilinear form

$$
\left\langle\left\langle\left(X_{1}, X_{2}\right),\left(Y_{1}, Y_{2}\right)\right\rangle\right\rangle=\left\langle X_{1}, Y_{1}\right\rangle-\left\langle X_{2}, Y_{2}\right\rangle \text {. }
$$

Put $b=L \mathfrak{b}, \bar{b}=L \overline{\mathfrak{b}}, n=L n, \bar{n}=L \overline{\mathfrak{n}}, h=L \mathfrak{h}$; let $\pi: b \rightarrow b / n, \bar{\pi}: \bar{b} \rightarrow \bar{b} / \bar{n}$ be the canonical homomorphisms; the quotient algebras $b / n, \bar{b} / \bar{n}$ may be canonically identified with $h$. Fix a linear operator $\theta \in E n d h$ which satisfies the following conditions:

(1) $\langle\theta X, \theta Y\rangle=\langle X, Y\rangle$ for any $X, Y \in h$

(2) $I-\theta$ is invertible. 
We shall assume, moreover, that $\theta$ extends to an automorphism of $L H$ which we denote by the same letter. Put

$$
g_{\theta}^{*}=\left\{\left(X_{+}, X_{-}\right) \in b \oplus \bar{b} \subset d ; \bar{\pi}\left(X_{-}\right)=\theta \circ \pi\left(X_{+}\right)\right\} ;
$$

let ${ }^{\delta} g \subset d$ be the diagonal subalgebra. The following assertion is well known.

Proposition 2.1. $\left(d,{ }^{\delta} g, g_{\theta}^{*}\right)$ is a Manin triple.

Thus we get a family of Lie bialgebras $\left(g, g_{\theta}^{*}\right)$ with common double $d=g \oplus g$ parametrized by $\theta \in E n d h$; all these bialgebras are factorizable. Let

$$
g=n \dot{+} h \dot{+} \bar{n}
$$

be the 'pointwise Bruhat decomposition' of the loop algebra. Let $P_{+}, P_{0}, P_{-}$be the corresponding projection operators which map $g$ onto $n, h, \bar{n}$, respectively. The classical r-matrix associated with $\left(g, g_{\theta}^{*}\right)$ is the kernel of the linear operator ${ }^{\theta} r_{+} \in$ $\operatorname{Hom}\left(g_{\theta}^{*}, g\right)$,

$$
{ }^{\theta} r_{+}=P_{+}+(I-\theta)^{-1} .
$$

Let us also set ${ }^{\theta} r_{-}:=-{ }^{\theta} r_{+}^{*}$; the classical Yang-Baxter identity implies that both ${ }^{\theta} r_{+}$ and ${ }^{\theta} r_{-}$are Lie algebra homomorphisms from $g_{\theta}^{*}$ into $g$. In the definition of the Poisson structures it is sometimes convenient to use their skew-symmetric combination,

$$
{ }^{\theta} r=\frac{1}{2}\left({ }^{\theta} r_{+}+{ }^{\theta} r_{-}\right)=\frac{1}{2}\left(P_{+}-P_{-}+\frac{1+\theta}{1-\theta} P_{0}\right) ;
$$

the 'perturbation term' in (2.4) is the Cayley transform of $\theta$,

$$
{ }^{\theta} r_{0}=\frac{1+\theta}{1-\theta} P_{0}
$$

The mappings ${ }^{\theta} r_{ \pm}$give rise to group homomorphisms ${ }^{\theta} r_{ \pm}:(L G)^{*} \rightarrow L G$ (which we denote by the same letters).

The double $d=g \oplus g$ has a natural structure of a factorizable Lie bialgebra associated with the Manin triple $\left(d,{ }^{\delta} g, g_{\theta}^{*}\right)$. Hence $\mathcal{D}=\mathcal{G} \times \mathcal{G}$ is a Poisson Lie group which contains both $\mathcal{G}$ and its dual group as Poisson subgroups. More precisely, let $\pi: L B \rightarrow L B / L N, \pi: L \bar{B} \rightarrow L \bar{B} / L \bar{N}$ be the canonical projections; the quotient groups $L B / L N, L \bar{B} / L \bar{N}$ may be canonically identified with $L H$. Let ${ }^{\delta} \mathcal{G} \subset \mathcal{G} \times \mathcal{G}$ be the diagonal subgroup and $\mathcal{G}^{*} \subset \mathcal{D}$ the subgroup defined by

$$
\mathcal{G}^{*}=\left\{\left(x_{+}, x_{-}\right) \in L B \times L \bar{B} ; \theta \circ \pi\left(x_{+}\right)=\bar{\pi}\left(x_{-}\right)\right\}
$$

Proposition 2.2. (i) ${ }^{\delta} \mathcal{G}, \mathcal{G}^{*} \subset \mathcal{D}$ are Poisson Lie subgroups with tangent Lie bialgebras $\left(g, g_{\theta}^{*}\right)$ and $\left(g_{\theta}^{*}, g\right)$, respectively. (ii) Almost all elements $x \in \mathcal{G}$ admit a factorization $x=x_{+} x_{-}^{-1}$ where $\left(x_{+}, x_{-}\right) \in \mathcal{G}^{*}$. 
We shall also need the related notion of twisted factorization.

Proposition 2.3. Suppose that the automorphism $\tau$ commutes with $\theta$. Then almost all elements $x \in \mathcal{G}$ admit a twisted factorization

$$
x=x_{+}^{\tau} x_{-}^{-1} \text {, where }\left(x_{+}, x_{-}\right) \in \mathcal{G}^{*} .
$$

Factorizations (2.6) and (2.7) are unique if we assume that both $x$ and $\left(x_{+}, x_{-}\right)$ are sufficiently close to the unit element.

2.2. Gauge covariant Poisson structures and reduction. Assume that $\theta \in$ End $h$ commutes with $\tau$. In that case the space $\mathcal{C}$ of q-difference connections admits a natural Poisson structure which is covariant with respect to the q-gauge action $\mathcal{G} \times \mathcal{C} \rightarrow \mathcal{C}$. We refer the reader to [14], [15] for its construction which is based on the notion of the twisted Heisenberg double. For any $\varphi \in C^{\infty}(\mathcal{C})$ we denote by $\nabla \varphi, \nabla^{\prime} \varphi$ its left and right gradients.

Theorem 2.4. (i) For any $\theta \in$ End h satisfying conditions (1), (2) the bracket

$$
\begin{array}{r}
\{\varphi, \psi\}_{\tau}=\left\langle{ }^{\theta} r(\nabla \varphi), \nabla \psi\right\rangle+\left\langle{ }^{\theta} r\left(\nabla^{\prime} \varphi\right), \nabla^{\prime} \psi\right\rangle \\
-\left\langle\tau \circ^{\theta} r_{+}\left(\nabla^{\prime} \varphi\right), Y\right\rangle-\left\langle{ }^{\theta} r_{-} \circ \tau^{-1}(\nabla \varphi), \nabla^{\prime} \psi\right\rangle,
\end{array}
$$

satisfies the Jacobi identity. (ii) Equip the space $\mathcal{C}$ of q-difference connections with the Poisson structure (2.8); then the q-gauge action defines a Poisson mapping $\mathcal{G} \times \mathcal{C} \rightarrow$ $\mathcal{C}$. (iii) The subgroup $\mathcal{N}=L N \subset \mathcal{G}$ is admissible and hence $\mathcal{N}$-invariant functions form a Poisson subalgebra in $C^{\infty}(\mathcal{C})$.

(We refer the reader to [9] for a general definition of admissible subgroups.) Below we shall need another formula for the Poisson bracket $\{,\}_{\tau}$ which is related to the twisted factorization in $\mathcal{G}$. Let $\varphi \in C^{\infty}(\mathcal{C})$; we define $Z_{\varphi} \in g$ by the following relation:

$$
r_{+} Z_{\varphi}-\tau^{-1} \cdot r_{-} Z_{\varphi}=\nabla^{\prime} \varphi
$$

Let $h \in \mathcal{C}$ be an element admitting twisted factorization, $h=h_{+}^{\tau} h_{-}^{-1}$; then

$$
\begin{aligned}
\{\varphi, \psi\}_{\tau}(h) & =\left\langle A d h_{+} \cdot \tau^{-1} \cdot Z_{\varphi}-A d h_{-} Z_{\varphi}, \nabla \psi\right\rangle \\
& -\left\langle\nabla \varphi, A d h_{+} \cdot \tau^{-1} \cdot Z_{\psi}-A d h_{-} Z_{\psi}\right\rangle
\end{aligned}
$$

We can now state our second main theorem. For $w \in W$ let $R_{w} \in$ End $L \mathfrak{h}$ be the linear operator acting in the loop algebra,

$$
\left(R_{w} H\right)(z)=A d w \cdot(H(z)) .
$$

Theorem 2.5. The quotient space $\mathcal{M}^{s} / \mathcal{N}=\mathcal{S}$ is a Poisson submanifold in $\mathcal{C} / \mathcal{N}$ if and only if the endomorphism $\theta$ is given by $\theta=R_{s} \cdot \tau$, where $s \in W$ is the Coxeter element. 
Notice that the Coxeter transformation satisfies conditions (1), (2) imposed above; since it preserves the root and weight lattices in $\mathfrak{h}$, it gives rise to an automorphism of $L H$. In the case when $\mathfrak{g}=\mathfrak{s l}(2)$, theorem 2.5 was proved in [9] (Theorem 2); in this case $R_{s}=-I d$.

For future reference let us write down explicitly the twisted factorization problem associated with the r-matrix ${ }^{\theta} r$.

Proposition 2.6. Assume that $\theta=R_{s} \cdot \tau$. Twisted factorization problem in the loop group $\mathcal{G}$ associated with the r-matrix ${ }^{\theta} r$ amounts to the relation

$$
x=y_{+} y_{-}^{-1}, y_{+} \in L B,, y_{-} \in L \bar{B}, \bar{\pi}\left(y_{-}\right)=s\left(\pi\left(y_{+}\right)\right) .
$$

We shall denote by $\mathcal{G}^{\prime} \subset \mathcal{G}$ the open subset consisting of elements admitting twisted factorization described in (2.10).

We shall now explicitly describe the kernel of the corresponding classical r-matrix. The relevant part of this kernel is the 'perturbation term' $r_{0}$ which was defined in (2.5). In the present situation we have

$$
r_{0}=\frac{I+R_{s} \cdot \tau}{I-R_{s} \cdot \tau} P_{0}
$$

Let $H_{p} \in \mathfrak{h}, p=1, \ldots, l$, be the eigenvectors of the Coxeter element, $\operatorname{Ads}\left(H_{p}\right)=$ $e^{\frac{2 \pi i k_{p}}{h}} H_{p}$ (here $h$ is the Coxeter number and $k_{1}, \ldots k_{l}$ are the exponents of $\mathfrak{g}$. There exists a permutation $\sigma$ of the set $\{1, \ldots l\}$ such that the basis $\left\{H_{\sigma p}\right\}$ is biorthogonal to $\left\{H_{p}\right\}$; we may assume that $\left\langle H_{p}, H_{\sigma p}\right\rangle=1$. Then

$$
\left(r_{0} X\right)(z)=\sum_{n=-\infty}^{\infty} \sum_{p=1}^{r} z^{n} \frac{1+q^{n} \exp \frac{2 \pi i k_{p}}{h}}{1-q^{n} \exp \frac{2 \pi i k_{p}}{h}}\left\langle X_{n}, H_{\sigma p}\right\rangle H_{p}, q \in \mathbb{C},|q|<1
$$

Note that the Lie bialgebra studied by Drinfeld in [3] (see also [10]) corresponds to the 'crystalline' limit $q \rightarrow 0$ in (2.12); in this case $r_{0}$ amounts to the Hilbert transform in $h$. It is convenient to write

$$
r_{0}(q, z)=\sum_{p=1}^{r} \psi_{p}(q, z) H_{p} \otimes H_{\sigma p}
$$

where

$$
\psi_{p}(q, z)=\sum_{n=-\infty}^{\infty} \frac{1+q^{n} \exp \frac{2 \pi i k_{p}}{h}}{1-q^{n} \exp \frac{2 \pi i k_{p}}{h}} z^{n} .
$$

Functions $\psi_{p}$ satisfy the q-difference equations,

$$
\psi_{p}(q, z)+\exp \frac{2 \pi i k_{p}}{h} \cdot \psi_{p}(q, q z)=\delta(z)-\exp \frac{2 \pi i k_{p}}{h} \cdot \delta(q z),
$$


where

$$
\delta(z)=\sum_{n=-\infty}^{\infty} z^{n} .
$$

2.3. Proof of Theorem 2.5. We briefly recall the geometric criterion that allows to check that a submanifold of a quotient Poisson manifold is itself a Poisson manifold. Let $M$ be a Poisson manifold, $\pi: M \rightarrow B$ a Poisson submersion. Hamiltonian vector fields $\xi_{\varphi}, \varphi \in \pi^{*} C^{\infty}(B)$, generate an integrable distribution $\mathfrak{H}_{\pi}$ in $T M$.

Proposition 2.7. Let $V \subset M$ be a submanifold; $W=\pi(V) \subset B$ is a Poisson submanifold if and only if $V$ is an integral manifold of $\mathfrak{H}_{\pi}$.

Assume that this condition holds true; let $\left.N_{V} \subset T^{*} M\right|_{V}$ be the conormal bundle of $V$; clearly, $\left.T^{*} V \simeq T^{*} M\right|_{V} / N_{V}$. Let $\varphi, \psi \in C^{\infty}(W)$; put $\varphi^{*}=\left.\pi^{*} \varphi\right|_{V}, \psi^{*}=\left.\pi^{*} \psi\right|_{V}$. Let $\overline{d \varphi},\left.\overline{d \psi} \in T^{*} M\right|_{V}$ be any representatives of $d \varphi^{*}, d \psi^{*} \in T^{*} V$. Let $P_{M} \in \Lambda^{2} V$ ect $M$ be the Poisson tensor.

Proposition 2.8. We have

$$
\left.\pi^{*}\{\varphi, \psi\}\right|_{V}=\left\langle P_{M}, \overline{d \varphi} \wedge \overline{d \psi}\right\rangle ;
$$

in particular, the r.h.s. does not depend on the choice of $\overline{d \varphi}, \overline{d \psi}$.

Let us now apply proposition 2.7 in the setting of theorem 2.5. It is sufficient to check that the Hamiltonian vector fields generated by $\mathcal{N}$-invariant functions on $\mathcal{C}$ are tangent to $\mathcal{M}^{s}$ if and only if $r_{0}$ is given by $(2.11)$. Let $\varphi \in C^{\infty}(\mathcal{C})^{\mathcal{N}}$; then $\varphi\left(v^{\tau} L\right)=\varphi(L v)$ for all $v \in \mathcal{N}$, and hence $Z:=\nabla \varphi-\tau \cdot \nabla^{\prime} \varphi \in b$. Since $\nabla^{\prime} \varphi(L)=$ Ad $L^{-1} \cdot \nabla \varphi(L)$, we rewrite the Poisson bracket on $\mathcal{C}$ in the following form:

$$
\{\varphi, \psi\}_{\tau}(L)=\left\langle r Z+Z-A d L \cdot r \cdot \tau^{-1} \cdot Z+A d L \cdot \tau^{-1} \cdot Z, \nabla \psi(L)\right\rangle ;
$$

thus in the left trivialization of $T \mathcal{C}$ the Hamiltonian field generated by $\varphi$ has the following form:

$$
\xi_{\varphi}(L)=r Z+Z-A d L \cdot r \cdot \tau^{-1} \cdot Z+A d L \cdot \tau^{-1} \cdot Z .
$$

Assume that $L \in \mathcal{M}^{s}, L=v s^{-1} u, v \in \mathcal{N}^{\prime}, u \in \mathcal{N}$. Put $Z=Z_{0}+Z_{+}, Z_{0} \in h, Z_{+} \in n$. Then

$$
\xi_{\varphi}(L)=r_{0} Z_{0}+Z_{0}+s^{-1} \tau^{-1} \cdot Z_{0}-s^{-1} \tau^{-1} \cdot r_{0} Z_{0}+X+A d\left(v \cdot s^{-1}\right) \cdot Y
$$

where $X \in n^{\prime}, Y \in n$. On the other hand, in the left trivialization of $T \mathcal{C}$ the tangent space $T_{L} \mathcal{M}^{f}$ is identified with $n^{\prime}+A d\left(v \cdot s^{-1}\right) \cdot n$. Hence $\xi_{\varphi}$ is tangent to $\mathcal{M}^{f}$ if and only its $h$-component vanishes, i.e., if

$$
r_{0}+I d+A d s^{-1} \tau^{-1}-A d s^{-1} \tau^{-1} r_{0}=0,
$$

which is equivalent to (2.11). 
2.4. Lattice $\mathcal{W}$-algebras. Let $\Gamma=\mathbb{Z} / N \mathbb{Z}$ be a finite periodic lattice. Set $G=$ $G^{\Gamma}, g=\mathfrak{g}^{\Gamma}$. Let $\tau$ be the automorphism of $\mathbb{G}$ induced by the cyclic permutation on $\Gamma, x_{i}^{\tau}=x_{i+1 \bmod N}$. We define lattice gauge transformations by

$$
g \cdot x=g^{\tau} x g^{-1}
$$

The definition of gauge covariant Poisson brackets on the space of q-difference connections has its obvious lattice counterpart. In an equally obvious way one may construct a class of Lie bialgebra structures on $g=\mathfrak{g}^{\Gamma}$ which is compatible with reduction over the unipotent subgroup $N=N^{\Gamma}$. Namely, let us consider the "pointwise Bruhat decomposition'

$$
g=n \dot{+} h \dot{+} \bar{n}, n=\mathfrak{n}^{\Gamma}, h=\mathfrak{h}^{\Gamma}, \bar{n}=\overline{\mathfrak{n}}^{\Gamma}
$$

and set

$$
{ }^{\theta} r=P_{+}-P_{-}+\frac{I+\theta}{I-\theta} P_{0}, \theta \in \text { Endh. }
$$

We shall omit the details and formulate only the lattice counterpart of the main theorems. Let as usual $s \in G$ be a Coxeter element.

Theorem 2.9. (i) The restriction of the gauge action to $N=N^{\Gamma}$ leaves the subset $M^{s}=N s^{-1} N$ invariant. (ii) The restricted action is free and $S=N^{\prime} s^{-1}, N^{\prime}=N^{\prime}$, is its cross-section.

We shall assume for simplicity that the lattice length $N$ is relatively prime with the Coxeter number.

Theorem 2.10. The quotient $M^{s} / N$ is a Poisson submanifold in the reduced space if and only if $\theta=R_{s} \cdot \tau$.

Remark. The condition on $\Gamma$ assures that $I-\theta$ is invertible; it is likely that reduction is possible even without this assumption, but this question needs further study.

For $G=S L(2)$ this reduction was studied in detail in [9], Section 6. It gives a discrete version of the Virasoro algebra, which is closely connected to the lattice Virasoro algebra of [5].

\section{The Cross-Section Theorem}

We shall prove the following assertion.

Theorem 3.1. For each $L \in \mathcal{N}^{\prime} s^{-1} \mathcal{N}$ there exists a unique element $n \in \mathcal{N}$ such that $n^{\tau} \cdot L \cdot s^{-1} \in \mathcal{N}^{\prime} s^{-1}$. 
Let $C_{h} \subset W$ be the cyclic subgroup generated by the Coxeter element. $C_{h}$ has exactly $l=\operatorname{rank} \mathfrak{g}$ different orbits in the root system $\Delta(\mathfrak{g}, \mathfrak{h})$. The proof depends on the structure of these orbits; for this reason we have to distinguish several cases. ${ }^{2}$

Proposition 3.2. The theorem is true for $\mathfrak{g}$ of type $/ A_{l}$.

An elementary proof which is based on the matrix algebra is given in [9]; below we present a different proof which uses only the properties of the corresponding root system.

Lemma 3.3. (i) Each orbit of $C_{h}$ in $\Delta(\mathfrak{g}, \mathfrak{h})$ consists of exactly $h$ elements; one can order these orbits in such a way that $k$-th orbit contains all positive roots of height $k$ and all negative roots of height $h-k$.

Put

$$
\mathfrak{n}_{k}=\bigoplus_{\left\{\alpha \in \Delta_{+}, h t \alpha=k\right\}} \mathfrak{n}_{\alpha}, N_{k}=\exp \mathfrak{n}_{k}, \mathcal{N}_{k}=L N_{k}
$$

For each $k$ we can choose $\alpha_{k} \in \Delta_{+}$in such a way that

$$
\mathfrak{n}_{k}=\bigoplus_{p=0}^{h-k-1} \mathfrak{n}_{s^{p}\left(\alpha_{k}\right)}
$$

Put $\mathfrak{n}_{k}^{p}=\mathfrak{n}_{s^{p}\left(\alpha_{k}\right)}, N_{k}^{p}=\exp \mathfrak{n}_{k}^{p}, \mathcal{N}_{k}^{p}=L N_{k}^{p}$.

Let $L=v \cdot s^{-1} \cdot u, v \in \mathcal{N}^{\prime}, u \in \mathcal{N}$; we must find $n \in \mathcal{N}$ such that

$$
n^{\tau} \cdot v \cdot s^{-1} \cdot u=v_{0} \cdot s^{-1} \cdot n .
$$

For any $n \in \mathcal{N}$ there exists a factorization

$$
n=n_{1} n_{2} \cdots n_{l}, \text { where } n_{k} \in \mathcal{N}_{k} ;
$$

moreover, each $n_{k}$ may be factorized as

$$
n_{k}=n_{k}^{0} n_{k}^{1} \cdots n_{k}^{h-k-1}, n_{k}^{p} \in \mathcal{N}_{k}^{p} .
$$

For any $n \in \mathcal{N}$ the element $n^{\tau} \cdot v \cdot s^{-1} \cdot u$ admits a representation

$$
n^{\tau} \cdot v \cdot s^{-1} \cdot u=\tilde{v} s^{-1} \tilde{u}, \tilde{v} \in \mathcal{N}^{\prime}, \tilde{u} \in \mathcal{N}
$$

let

$$
\tilde{u}=\prod_{k=1}^{l} \prod_{p=0}^{\overrightarrow{h-k-1}} \tilde{u}_{k}^{p}, \tilde{u}_{k}^{p} \in \mathcal{N}_{k}^{p},
$$

be the corresponding factorization of $\tilde{u}$. For $x \in G$ we write $s(x):=s \cdot x \cdot s^{-1}$ (this notation will be frequently used in the sequel).

\footnotetext{
${ }^{2}$ The proofs given below do not apply when $\mathfrak{g}$ is a simple Lie algebra of type $E_{6}$.
} 
Lemma 3.4. We have $\tilde{u}_{k}^{p}=\tau \cdot s\left(n_{k}^{p-1}\right) V_{k}^{p}$, where the factors $V_{k}^{p} \in \mathcal{N}_{k}^{p}$ depend only on $u, v$ and on $n_{j}^{q}$ with $j<k$.

Assume now that $n$ satisfies (3.1); then we have $\tilde{v}=v_{0}, \tilde{u}=n$. This leads to the following relations:

$$
\tau \cdot s\left(n_{k}^{p-1}\right) V_{k}^{p}=n_{k}^{p}
$$

where we set formally $n_{k}^{-1}=1$.

Proposition 3.5. The system (3.2) may be solved recursively starting with $k=1$, $p=0$.

Clearly, the solution is unique. This concludes the proof for $\mathfrak{g}$ of type $A_{l}$.

Let now $\mathfrak{g}$ be a simple Lie algebra of type other than $A_{l}$ and $E_{6}, l$ its rank.

Lemma 3.6. (i) The Coxeter number $h(\mathfrak{g})$ is even. (ii) Each orbit of $C_{h}$ in $\Delta(\mathfrak{g}, \mathfrak{h})$ consists of exactly $h$ elements and contains an equal number of positive and negative roots. (iii) Put

$$
\Delta_{+}^{p}=\left\{\alpha \in \Delta_{+} ; s^{-p} \cdot \alpha \notin \Delta_{+}\right\}, \mathfrak{n}^{p}=\bigoplus_{\alpha \in \Delta_{+}^{p}} \mathbb{C} \cdot e_{\alpha} ;
$$

then $\mathfrak{n}^{p} \subset \mathfrak{n}$ is an abelian subalgebra, $\operatorname{dim} \mathfrak{n}^{p}=l$.

When $\mathfrak{g}$ is not of type $D_{2 k+1}$ this assertion follows from [2] (Chapter 6, no 1.11, prop. 33 and Chapter 3, no 6.2, corr. 3). For $\mathfrak{g}$ of type $D_{2 k+1}$ it may be checked directly.

Put $N^{p}=\exp \mathfrak{n}^{p}$; let $\mathcal{N}^{p}$ be the corresponding subgroup of the loop group $\mathcal{G}$. Let $L=v \cdot s^{-1} \cdot u, v \in \mathcal{N}^{\prime}, u \in \mathcal{N}$; we must find $n \in \mathcal{N}$ such that

$$
v \cdot s^{-1} \cdot u=n^{\tau} \cdot v_{0} \cdot s^{-1} \cdot n^{-1} \text {. }
$$

Put

$$
n=n_{1} n_{2} \cdots n_{\frac{h}{2}}, n_{p} \in \mathcal{N}_{p}
$$

the elements $n_{p}$ will be determined recursively. Put $s^{-1}(w)=s^{-1} \cdot w \cdot s, w \in N$. We have

$$
v \cdot s^{-1}(u)=\left(\tau \cdot \vec{\prod} n_{p}\right) \cdot v_{0} \cdot s^{-1}\left(\overleftarrow{\prod} n_{p}^{-1}\right)
$$

We shall say that an element $x \in \mathcal{G}$ is in the big cell in $\mathcal{G}$ if, for all values of the argument $z$, the value $x(z)$ is in the big Bruhat cell $B \bar{N} \subset G$.

Lemma 3.7. $v \cdot s^{-1}(u)$ is in the big cell in $\mathcal{G}$ and admits a factorization

$$
v \cdot s^{-1}(u)=x_{+}^{1} \cdot x_{-}^{1}, x_{+}^{1} \in \mathcal{N}, x_{-}^{1} \in \overline{\mathcal{N}}
$$


Indeed, let $u=u_{h / 2} u_{h / 2-1} \cdots u_{1}, u_{p} \in \mathcal{N}^{p}$, be a similar decomposition of $u$; then we have simply $x_{-}=s^{-1}\left(u_{1}\right)$. (It is clear that $x_{+}^{1} \in \mathcal{B}$ actually does not have an $\mathcal{H}$-component and so belongs to $\mathcal{N}$.)

A comparison of the r.h.s in (3.4) with the Bruhat decomposition of the 1.h.s. immediately yields that the first factor in (3.3) is given by $n_{1}=s\left(x_{-}\right)^{-1}$.

Assume that $n_{1}, n_{2}, \ldots, n_{k-1}$ are already computed. Put

$$
m_{k}=n_{1} n_{2} \cdots n_{k-1}
$$

and consider the element

$$
L^{k}:=s^{-k+1}\left(\tau\left(m_{k}^{-1}\right) \cdot\left(v \cdot s^{-1}(u)\right) \cdot s^{-1}\left(m_{k}\right)\right) .
$$

Lemma 3.8. $L^{k}$ is in the big cell in $\mathcal{G}$ and admits a factorization

$$
L^{k}=x_{+}^{k} x_{-}^{k}, x_{+}^{k} \in \mathcal{N}, x_{-}^{k} \in \overline{\mathcal{N}} .
$$

The elements $x_{ \pm}^{k}$ are computed recursively from the known quantities. By applying a similar transform to the r.h.s. of (3.4) we get

$$
\begin{aligned}
L^{k}= & s^{-k+1}\left(\tau\left(m_{k}^{-1}\right) \cdot\left(\tau \cdot \prod_{p} n_{p}\right) \cdot v_{0} \cdot s^{-1}\left(\overleftarrow{\prod}_{p} n_{p}^{-1}\right) \cdot s^{-1}\left(m_{k}\right)\right)= \\
& s^{-k+1}\left(\left(\tau \cdot \overrightarrow{\prod_{p \geq k}} n_{p}\right) v_{0}\right) \cdot s^{-k}\left(\overleftarrow{\prod_{p \geq k+1}} n_{p}^{-1}\right) \cdot s^{-k}\left(n_{k}^{-1}\right) .
\end{aligned}
$$

A comparison of (3.7) and (3.6) yields $x_{-}^{k}=s^{-k}\left(n_{k}^{-1}\right)$; hence $n_{k}=s^{k}\left(x_{-}^{k}\right)$, which concludes the induction.

\section{Generalized Miura Transform}

Our construction of the Miura transform for q-difference operators may be regarded as a nonlinear version of the corresponding construction for differential operators, due to Drinfeld and Sokolov [4]. Recall that the space of abstract differential operators associated with a given semisimple Lie algebra $\mathfrak{g}$ is realized as the quotient space of the affine manifold $\mathcal{M}^{f}=f+L \mathfrak{b} \subset L \mathfrak{g}$ (the translate of $L \mathfrak{b}$ by a fixed principal nilpotent element $f \in \mathfrak{n}$ ) over the gauge action of $L N$. The cross-section theorem of Drinfeld and Sokolov provides a global model $\mathcal{S}$ for this quotient space. It is easy to see that the affine submanifold $f+L \mathfrak{h} \subset f+L \mathfrak{b}$ is a local cross-section of the gauge action $L N \times \mathcal{M}^{f} \rightarrow \mathcal{M}^{f}$ (i.e., the orbits of $L N$ are transversal to $f+L \mathfrak{h}$ ) and hence $f+L \mathfrak{h}$ provides a local model of the same quotient space. Thus we get a Poisson structure on $f+L \mathfrak{h}$ and a Poisson mapping $f+L \mathfrak{h} \rightarrow \mathcal{S}$. The computation of the induced Poisson structure on $f+L \mathfrak{h}$ follows the general prescription of Dirac (see, e.g., [6]), but is in fact greatly simplified, since all correction terms in the Dirac formula identically vanish. One may notice that the affine manifold $f+L \mathfrak{h} \subset \mathcal{M}^{f}$ is 
the intersection of the level surfaces of two moment maps associated with the gauge actions of the opposite triangular subgroups $L N$ and $L \bar{N}$; it is this symmetry between $L N$ and $L \bar{N}$ that accounts for cancellations in the Dirac formula. The situation in the nonlinear case is exactly similar.

We pass to the formal description of our construction. Let $\bar{B} \subset G$ be the opposite Borel subgroup, $\bar{N} \subset \bar{B}$ its nilradical, $\overline{\mathcal{B}}=L \bar{B}, \overline{\mathcal{N}}=L \bar{N}$. Let us consider the Poisson reduction of the space $\mathcal{C}$ of q-difference connections over the action of the opposite gauge group $\overline{\mathcal{N}}$. We equip $\mathcal{C}$ with the Poisson structure $(2.8)$, where the choice of $\theta$ may be arbitrary.

Proposition 4.1. (i) The q-gauge action $\overline{\mathcal{N}} \times \mathcal{C} \rightarrow \mathcal{C}$ leaves $\overline{\mathcal{B}} \subset \mathcal{C}$ invariant. (ii) Hamiltonian vector fields on $\mathcal{C}$ generated by gauge invariant functions $\varphi \in C^{\infty}(\mathcal{C})^{\overline{\mathcal{N}}}$ are tangent to $\overline{\mathcal{B}} \subset \mathcal{C}$.

Corollary 4.2. $\overline{\mathcal{B}} / \overline{\mathcal{N}} \subset \mathcal{C} / \overline{\mathcal{N}}$ is a Poisson submanifold.

Remark. Heuristically, the submanifold $\overline{\mathcal{B}} \subset \mathcal{C}$ corresponds to reduction at the 'zero level' of the moment, hence the constraints are automatically of the first class.

We shall now define an embedding $i: \mathcal{H} \rightarrow \mathcal{M}^{s} \cap \overline{\mathcal{B}}$ into the intersection of two 'level surfaces'.

Let $w_{0} \in W$ be the longest element; let $\pi \in$ Aut $\Delta_{+}$be the automorphism defined by $\pi(\alpha)=-w_{0} \cdot \alpha, \alpha \in \Delta_{+}$. Let $N_{i} \subset N$ be the 1-parameter subgroup generated by the root vector $e_{\pi\left(\alpha_{i}\right)}, \alpha_{i} \in P$. Choose an element $u_{i} \in N_{i}, u_{i} \neq 1$.

Lemma 4.3. $[16] w_{0} u_{i} w_{0}^{-1} \in B s_{i} B$.

We may choose $u_{i}$ in such a way that $w_{0} u_{i} w_{0}^{-1} \in N s_{i} N$. Put $x=u_{l} u_{l-1} \ldots u_{1}$; then $f:=w_{0} x w_{0}^{-1} \in N s^{-1} N \cap \bar{N}$.

Remark. The choice of $u_{i}$ is not unique, however this non-uniqueness does not affect the arguments below. $\square$

Define the immersion $i: H \rightarrow G: x \longmapsto x \cdot f \cdot s\left(x^{-1}\right)$.

Proposition 4.4. $i(H) \subset N s^{-1} N \cap \bar{B}$.

Remark. By dimension count it is easy to see that $i(H)$ is open in $N s^{-1} N \cap \bar{B}$. For $G=S L(n)$ we have simply $i(H)=N s^{-1} N \cap \bar{B}$; it seems plausible that this is true in the general case as well.

We define the corresponding embedding $i: \mathcal{H} \rightarrow \mathcal{G}$ for loop groups associated with $H, G$ by the same formula. Clearly, $i(\mathcal{H}) \subset \mathcal{M}^{s} \cap \overline{\mathcal{B}}$.

Proposition 4.5. $i(\mathcal{H})$ is a local cross-section of the gauge actions $\mathcal{N} \times \mathcal{M}^{s} \rightarrow$ $\mathcal{M}^{s}, \overline{\mathcal{N}} \times \overline{\mathcal{B}} \rightarrow \overline{\mathcal{B}}$ 
In other words, gauge orbits of $\mathcal{N}, \overline{\mathcal{N}}$ are transversal to $i(\mathcal{H}) \subset \mathcal{M}^{s} \cap \overline{\mathcal{B}}$.

Let us now assume that the Poisson structure on the space of q-difference connections is the one described in theorem 2.5. We may consider $i(\mathcal{H})$ as a (local) model of the reduced space $\mathcal{M}^{s} / \mathcal{N}$ obtained by 'fixing the gauge' by means of the 'subsidiary condition' $L \in \overline{\mathcal{B}}$, or, alternatively, as a model of $\overline{\mathcal{B}} / \overline{\mathcal{N}}$ obtained by choosing the subsidiary condition $L \in \mathcal{M}^{s}$. The choice of $r_{0}$ assures that both the 'constraints' and the 'subsidiary conditions' are of the first class. The reduced Poisson structure on $i(\mathcal{H})$ may be expressed in terms of the Dirac bracket. As it appears, it is possible to avoid the actual computation of the 'correction terms'. We shall prove the following assertion.

Proposition 4.6. The quotient Poisson structure on $i(\mathcal{H})$ is given by

$$
\{\varphi, \psi\}_{i(\mathcal{H})}=\left\langle\tilde{P}_{\mathcal{H}} \nabla \varphi, \nabla \psi\right\rangle, \tilde{P}_{\mathcal{H}}=\frac{(I d-\tau)\left(I d-R_{s}\right)}{I d-R_{s} \cdot \tau}
$$

It will be convenient to introduce another parametrization of the Cartan subgroup which is related to the twisted factorization problem $(2.10)$ in $\mathcal{G}$.

Lemma 4.7. $\overline{\mathcal{B}} \subset \mathcal{G}^{\prime}$

Proof. The twisted factorization problem in $\overline{\mathcal{B}}$ (cf. (2.10) amounts to the relation

$$
\bar{b}=x_{+} \cdot x_{-}^{-1} n_{-}^{-1} \text {, where } x_{+} \in \mathcal{H}, x_{-} \in \mathcal{H}, n_{-} \in \overline{\mathcal{N}} \text { and } x_{-}=s\left(x_{+}\right),
$$

or, equivalently,

$$
\bar{b}=x \cdot s(x)^{-1} n_{-}^{-1} .
$$

The same assertion of course holds true for $\mathcal{H} \subset \overline{\mathcal{B}}$; in that case we have $n_{-}=1$. Let $\pi: B \rightarrow H$ be the projection map which assigns to $h \in B$ the element $x \in H$ satisfying (4.2).

Lemma 4.8. Let $H \subset G$ be the Cartan subgroup. The mapping $p: H \rightarrow H: x \longmapsto$ $x \cdot s(x)^{-1}$ is an immersion.

Put

$$
P_{\mathcal{H}}=\frac{R_{s} \cdot(\tau-I d)}{\left(I d-R_{s}\right)\left(I d-R_{s} \cdot \tau\right)}
$$

and define the Poisson bracket on $\mathcal{H}$ by

$$
\{\varphi, \psi\}_{\mathcal{H}}=\left\langle P_{\mathcal{H}} D \varphi, D \psi\right\rangle \text {. }
$$

Lemma 4.9. $p:\left(\mathcal{H},\{,\}_{P_{\mathcal{H}}}\right) \rightarrow\left(\mathcal{H},\{,\}_{i(\mathcal{H})}\right)$ is a Poisson mapping. 
Hence to prove proposition 4.6 we may use the Poisson bracket (4.4) instead of (4.1). Let $\varphi, \psi \in C^{\infty}(\mathcal{H})$. Let $\varphi^{*}=\varphi \circ \pi,, \psi^{*}=\psi \circ \pi \in C^{\infty}(\overline{\mathcal{B}})$ be their lifts to $\overline{\mathcal{B}}$ defined via the twisted factorization map. (In other words,

$$
\left.\varphi^{*}(\bar{b})=\varphi(x), \text { where } \bar{b}=x \cdot s(x)^{-1} n_{-}^{-1}, x \in \mathcal{H} .\right)
$$

In the right trivialization of the cotangent bundle of $\overline{\mathcal{B}}$ the differential $d \varphi^{*}(h) \in b^{*}$ of $\varphi^{*}$ is given by

$$
d \varphi^{*}(h)=-\tau^{-1} r_{-} \nabla \varphi
$$

where $\nabla \varphi \in h$ is the right invariant differential of $\varphi$ evaluated at $x=\pi(h)$, and similarly for $\psi^{*}$. The standard embedding $b^{*} \subset g$ allows to regard $d \varphi^{*}(h)$ as an element of $g$. To compute the Poisson bracket $\{\varphi, \psi\}$ we may apply proposition 2.8. We have

$$
\{\varphi, \psi\}(\pi(h))=\left\langle P_{\mathcal{C}}, d \varphi^{*}(h) \wedge d \psi^{*}(h)\right\rangle .
$$

Using formula (2.9) and inserting the expression (4.5) for the differentials we get (4.4).

Let $c: \mathcal{M}^{s} \rightarrow \mathcal{S}$ be the canonical mapping which assigns to each $L \in \mathcal{M}^{s}$ the unique element $L^{0} \in \mathcal{S}$ lying in the same $\mathcal{N}$-orbit. The generalized Miura transform $m$ is defined by $m=c \circ i: \mathcal{H} \rightarrow \mathcal{S}$.

Theorem 4.10. The generalized Miura transform is a Poisson mapping.

The Poisson structure in $\mathcal{H}$ is given by (4.4); the Poisson structure in the target space is the reduced Poisson structure described in theorem 2.5.The proof immediately follows from the fact that $i(\mathcal{H})$ and $\mathcal{S}$ are different models of the quotient space $\mathcal{M}^{s} / \mathcal{N}$

Note that for $G=S L(2)$ our construction of the Miura transform coincides with the one described in $[9]$.

\section{THe $S L(n)$ CASE}

Our aim in this section is to compare the Poisson structures arising via the qDrinfeld-Sokolov reduction with the results in [8]. (The case of $n=2$ has been discussed in detail in [9]. Our analysis for general $n$ is parallel to that of [9], Section 3, though our conventions are slightly different.) To begin with, let us list the standard facts concerning the structure of $S L(n)$. We keep to the choice of order in the root system of $\mathfrak{s} l(n)$ made in section 1 , that is, positive root vectors correspond to lower triangular matrices. We order simple roots in such a way that the Coxeter element $s=s_{1} s_{2} \cdots s_{n-1}$ is acting on the Cartan subalgebra $\mathfrak{h}$ as a cyclic permutation,

$$
s^{-1} \cdot \operatorname{diag}\left(H_{1}, H_{2}, \ldots, H_{n}\right)=\operatorname{diag}\left(H_{n}, H_{1}, \ldots, H_{n-1}\right)
$$


its representative in $G=S L(n)$ is given by

$$
s^{-1}=\left(\begin{array}{ccccc}
0 & -1 & 0 & \cdots & 0 \\
0 & 0 & -1 & \cdots & 0 \\
\cdots & \cdots & \cdots & \cdots & \cdots \\
0 & 0 & 0 & \cdots & -1 \\
1 & 0 & 0 & \cdots & 0
\end{array}\right)
$$

The automorphism $\pi=-w_{0}$ of $\mathfrak{h}$ is given by

$$
\pi \cdot \operatorname{diag}\left(H_{1}, H_{2}, \ldots, H_{n}\right)=\operatorname{diag}\left(-H_{n},-H_{n-1}, \ldots,-H_{1}\right) .
$$

We may choose the unipotent elements $u_{i}, i=1,2, \ldots, n-1$, in such a way that the principal nilpotent element $f$ constructed in lemma 4.3 is given by

$$
f=\left(\begin{array}{ccccc}
1 & -1 & 0 & \cdots & 0 \\
0 & 1 & -1 & \cdots & 0 \\
\cdots & \cdots & \cdots & \cdots & \cdots \\
0 & 0 & 0 & \cdots & -1 \\
0 & 0 & 0 & \cdots & 1
\end{array}\right)
$$

the manifold $\mathcal{M}^{s}$ consists of matrices of the form

$$
L=\left(\begin{array}{ccccc}
* & -1 & 0 & \cdots & 0 \\
* & * & -1 & \cdots & 0 \\
\cdots & \cdots & \cdots & \cdots & \cdots \\
* & * & * & \cdots & -1 \\
* & * & * & \cdots & *
\end{array}\right)
$$

Let $x=\operatorname{diag}\left(x_{1}, x_{2}, \ldots, x_{n}\right)$; the embedding $i: \mathcal{H} \rightarrow \mathcal{M}^{s}$ defined in proposition 4.3 is given by

$$
i(x):=\Lambda=\left(\begin{array}{ccccc}
x_{1} x_{n}^{-1} & -1 & 0 & \cdots & 0 \\
0 & x_{2} x_{1}^{-1} & -1 & \cdots & 0 \\
\cdots & \cdots & \cdots & \cdots & \cdots \\
0 & 0 & 0 & \cdots & -1 \\
0 & 0 & 0 & \cdots & x_{n} x_{n-1}^{-1}
\end{array}\right)
$$

It is convenient to introduce affine coordinates on $i(\mathcal{H})$ in the following way:

$$
\Lambda(z)=\left(\begin{array}{ccccc}
\Lambda_{1}(z) & -1 & 0 & \cdots & 0 \\
0 & \Lambda_{2}(q z) & -1 & \cdots & 0 \\
\cdots & \cdots & \cdots & \cdots & \cdots \\
0 & 0 & 0 & \cdots & -1 \\
0 & 0 & 0 & \cdots & \Lambda_{n}\left(q^{n-1} z\right)
\end{array}\right)
$$


Let $L_{c a n}=m(\Lambda) \in \mathcal{S}$ be the canonical form of $\Lambda$,

$$
L_{c a n}=\left(\begin{array}{ccccc}
0 & -1 & 0 & \cdots & 0 \\
0 & 0 & -1 & \cdots & 0 \\
\cdots & \cdots & \cdots & \cdots & \cdots \\
0 & 0 & 0 & \cdots & -1 \\
1 & u_{1}(z) & u_{2}(z) & \cdots & u_{n-1}(z)
\end{array}\right)
$$

we put $u_{p}(z):=(-1)^{n-p-1} s_{n-p}\left(q^{n-p} z\right)$.

Proposition 5.1. ([9], Lemma 2) We have

$$
s_{p}(z)=\sum_{1 \leq j_{1}<j_{2}<\ldots<j_{p} \leq n} \Lambda_{j_{1}}(z) \Lambda_{j_{2}}(q z) \cdots \Lambda_{j_{p}}\left(q^{p-1} z\right) .
$$

Proposition 5.2. The Poisson bracket on $\mathcal{H}$ is given by

$$
\begin{gathered}
\left\{\Lambda_{p}(z), \Lambda_{p}(w)\right\}=\sum_{m=-\infty}^{\infty} \frac{\left(1-q^{m}\right)\left(1-q^{m(n-1)}\right)}{1-q^{n m}}\left(\frac{z}{w}\right)^{m} \Lambda_{p}(z) \Lambda_{p}(w), \\
\left\{\Lambda_{p}(z), \Lambda_{s}(w)\right\}=\sum_{n=-\infty}^{\infty} \frac{\left(1-q^{m}\right)\left(1-q^{-m}\right)}{1-q^{n m}}\left(\frac{z}{w}\right)^{m} \Lambda_{p}(z) \Lambda_{s}(w), s>p .
\end{gathered}
$$

Proof. Let $\omega=\exp \frac{2 \pi i}{n}$ be the primitive root of unity. The eigenvectors of $s$ in $\mathfrak{h}$ are

$$
e_{k}=\operatorname{diag}\left(1, \omega^{-k}, \ldots, \omega^{-(n-1) k}\right), s \cdot e_{k}=\omega^{k} e_{k}, k=1, \ldots, n-1 .
$$

The kernel of the Poisson operator $\tilde{P}_{\mathcal{H}}=\frac{(I d-\tau)\left(I d-R_{s}\right)}{I d-\tau \cdot R_{s}}$ is given by the formal Laurent series

$$
\sum_{m=-\infty}^{\infty} \sum_{k=1}^{n-1} \frac{1}{n} \frac{\left(1-q^{m}\right)\left(1-\omega^{k}\right)}{1-q^{n} \omega^{k}}\left(\frac{z}{w}\right)^{m} e_{k} \otimes e_{n-k} .
$$

We have

$$
\begin{aligned}
& =\sum_{m=-\infty}^{\infty} \sum_{k=1}^{n-1} \frac{1}{n} \frac{\left(1-q^{m}\right)\left(1-\omega^{k}\right)}{1-q^{m} \omega^{k}}\left(\frac{z}{w}\right)^{m} q^{m(s-p)}\left(e_{k} \cdot \Lambda(z)\right)_{p p} \cdot\left(e_{n-k} \cdot \Lambda(w)\right)_{s s} \\
& =\sum_{m=-\infty}^{\infty} \sum_{k=1}^{n-1} \frac{1}{n} \frac{\left(1-q^{m}\right)\left(1-\omega^{k}\right)}{1-q^{m} \omega^{k}}\left(\frac{z}{w}\right)^{m} q^{m(s-p)} \omega^{k(s-p)} \Lambda_{p}(z) \Lambda_{s}(w) .
\end{aligned}
$$

Observe that

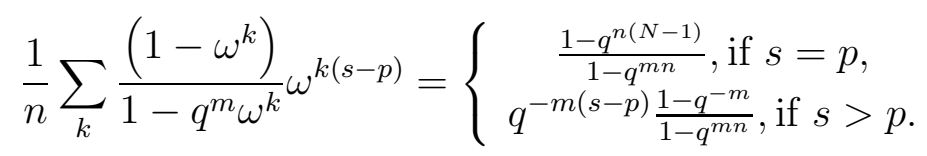

Substituting (5.4) into (5.3), we get (5.2). 
Formula (5.1) coincides with the $q$-deformed Miura transformation defined in [8]. Formula (5.2) coincides with the Poisson bracket on $\Lambda_{i}(z)$ 's derived in [8]. Therefore in the case of $\mathfrak{s l}(n)$ the Poisson algebra obtained by the difference Drinfeld-Sokolov reduction coincides with the $q$-deformed $\mathcal{W}$-algebra introduced in [8].

\section{REFERENCES}

1. Belavin A.A., Drinfeld V.G., Solutions of the classical Yang-Baxter equation for simple Lie algebras, Funct. Anal. Appl., 16 (1981), 159-80.

2. Bourbaki N. Groupes et algèbres de Lie. Paris, Hermann, 1968.

3. Drinfeld V.G., A new realization of Yangians and quantized affine algebras, Sov. Math. Dokl. 36 (1988).

4. Drinfeld V.G., Sokolov V.V., Lie algebras and equations of Korteweg-de Vries type, Sov. Math. Dokl. 23 (1981), 457-62; J. Sov. Math. 30 (1985), 1975-2035.

5. Faddeev, L.D., Volkov, A.Yu., Abelian current algebras and the Virasoro algebra on the lattice, Phys. Lett. B315 (1993), 311-8.

6. Flato, M., Lichnerowicz, A., Sternheimer, D., Deformation of Poisson brackets, Dirac brackets and applications, J. Math. Phys. 17 (1976), 1754.

7. Frenkel E., Affine Kac-Moody algebras at the critical level and quantum Drinfeld-Sokolov reduction. PhD Thesis, Harvard University, 1991.

8. Frenkel, E., Reshetikhin, N., Quantum affine algebras and deformations of the Virasoro algebra and $W$-algebras, Comm. Math. Phys. 178 (1996), 237-264; q-alg/9505025.

9. Frenkel, E., Reshetikhin, N., Semenov-Tian-Shansky M..A., Drinfeld-Sokolov reduction for difference operators and deformations of $W$-algebras I. The case of Virasoro algebra. q-alg/9704011

10. Khoroshkin S.M., Tolstoy V.N. , On Drinfeld's realization of quantum affine algebras, J. Geom. Phys. 11 (1993), 445-52.

11. Kostant B. (1978). On Whittaker vectors and representation theory, Inventiones Math. 48, 101-184.

12. Kostant B., The solution to a generalized Toda lattice and representation theory, Adv. Math. 34 (1980), 13-53.

13. Semenov-Tian-Shansky M.A., What is a classical r-matrix, Funct. Anal. Appl., 17 (1983), 17-33.

14. Semenov-Tian-Shansky M.A., Dressing action transformations and Poisson - Lie group actions. Publ. Math. RIMS, 21 (1985), 1237-1260.

15. Semenov-Tian-Shansky M.A. Poisson Lie groups, quantum duality principle and the quantum double. Contemporary Math., 175, 219-248.

16. Steinberg R., Regular elements of semisimple algebraic Lie groups, Publ. Math. I.H.E.S., 25 (1965), 49-80.

Université de Bourgogne, Dijon, France,, and Steklov Mathematical Institute, St. Petersburg,

Institut of Theoretical Physics, Uppsala University, Uppsala, Sweden, and Steklov Mathematical Institute, St.Petersburg 